\title{
Automatic calibration of groundwater models using global optimization techniques
}

\author{
D. P. SOLOMATINE, Y. B. DIBIKE
}

International Institute for Infrastructural, Hydraulic and Environmental Engineering (IHE), PO Box 3015, 2601 DA Delft, The Netherlands

e-mail: sol@ihe.nl

\author{
N. KUKURIC \\ The Netherlands Institute of Applied Geoscience TNO, PO Box 6012, 2600 JA Delft, \\ The Netherlands
}

\begin{abstract}
The problem of a groundwater model calibration is posed as a multiextremum (global) optimization problem, rather than the more widely considered single-extremum (local) optimization problem. Several algorithms of randomized search incorporated in the global optimization tool GLOBE are considered (including the canonical genetic algorithm and more recently developed adaptive cluster covering), and applied to the calibration of the groundwater model TRIWACO. The results show the usefulness of global optimization algorithms in the automatic calibration of even complex models having considerable running times.
\end{abstract}

\section{Calage automatique de modèles d'écoulement souterrain utilisant des techniques d'optimisation globales}

Résumé Par opposition à l'approche classique qui considère le problème du calage des modèles d'écoulement souterrain comme un problème d'optimisation à un extremum (optimisation locale), nous posons ce problème ici en termes d'optimisation à extremums multiples (optimisation globale, OG). L'application de divers algorithmes de recherche aléatoire (recherche aléatoire contrôlée, algorithme génétique canonique et couverture adaptative) au modèle d'écoulement souterrain TRIWACO montre l'intérêt de l'utilisation des algorithmes OG pour le calage de modèles qui, en raison de leur complexité, exigent des temps de calcul considérables.

\section{INTRODUCTION}

At present, important shifts in the understanding of the importance of automating one of the crucial phases of model construction-model calibration-can be observed. More and more practitioners do not want to rely solely upon the time consuming "trialand-error" approach, and show interest in using more systematic procedures for calibration.

The possibilities of using optimization methods in hydrological model calibration have been demonstrated, e.g. by Dawdy \& O’Donnell (1965), Johnston \& Pilgrim (1976), Hendrikson et al. (1988) (surface water hydrology), and for groundwater models by Yeh (1986), Carrera \& Neuman (1986), Carrera (1988), Peck et al. (1988) and Olsthoorn (1995). Most publications on groundwater modelling omit to consider an important problem - the number of minima of the model error function (e.g. Model Calibration, 1997). Analysis of the most popular commercial tools for the automatic calibration of groundwater models (MODFLOP, PEST, MODINV-Scientific Software Group, 1996) also shows that they use variations of the Gauss-Newton 
method, which is a method of local search and is incapable of finding a global minimum. The latest research in calibration of groundwater models has concentrated on applications of various methods of single-extreme optimization (e.g. Olsthoorn, 1995), error function type (Zaadnoordijk \& Stein, 1997) and parameter sensitivity (Hemker, 1997).

However, there is no evidence that the error function of a numerical model has only one minimum - rather, this is normally a multi-extreme function. In surface water hydrology, the problem of calibration is treated more and more as a multi-extreme problem (Wang, 1991; Sorooshian et al., 1993; Solomatine, 1995; Franchini \& Galeati, 1997; Kuczera, 1997). This may be attributed to the fact that the models being calibrated are simpler than groundwater models, and their running times are much less.

The purpose of this article is to consider the possibilities of using global, or multiextreme techniques in automated calibration of numerical groundwater models. Applicability of these techniques is demonstrated with a real case study. A global optimization tool GLOBE (Solomatine 1998, 1999) is applied to calibrate a numerical groundwater model developed with the use of the modelling package TRIWACO (1992). The performance of a new method of global optimization, adaptive cluster covering, is compared with another global optimization technique-the genetic algorithm.

\section{THE PROBLEM OF MODEL CALIBRATION}

The objective of calibration (parameter optimization) of any model of a physical system is to identify the values of some parameters in the model which are not known a priori. This is achieved by feeding the model with input data, and comparing the computed output variables to the variable values measured in the physical system. Their difference should be minimized by solving an optimization problem, with independent variables being the unknown model parameters.

In the trial-and-error calibration of groundwater models, the model parameters such as hydraulic conductivity and specific storage are assigned to each node and the model calculates values of piezometric heads. The calculated heads are then compared with measured values and the model parameters are adjusted according to a modeller's judgement, in order to provide a better match. The calibration is repeated until an adequate match (with acceptable error) is obtained.

Given the vector $\mathbf{O B S}_{t}$ of observed output variables values, and the corresponding vector $\mathbf{M O D}_{t}$ of the modelled values at time moments $t=1, \ldots, T$, one of the widely used evaluation criteria for the discrepancy (error) between the model results and observations (in other words, the goodness of fit of the model) is:

$$
E=\frac{1}{T} \sum_{t-1}^{T}\left|\mathbf{O B S}_{t}-\mathbf{M O D}_{t}\right|^{\gamma}
$$

where $\gamma$ is taken between 1 and 2 , and $w_{t}$ is the weight associated with particular moments of time (for $\gamma=2$ and $w_{t}=1, E$ is the mean squared error). Sorooshian et al. (1993) and Pintér (1995) report the high degree of nonlinearity, and often nonsmoothness of such functions. The weighted accumulated deviation can be used also.

The problem of calibration may be formulated in different ways, for example as an optimal parameter estimation, or data assimilation, etc., but the essence of the problem 
remains the same-solving the problem of the model error minimization. If the problem is formulated as a single-extreme minimization problem, and certain assumptions are made about the model structure and/or model error formulation, then there are techniques available that allow the solution to be accelerated, e.g. those of adjoint modelling (Van den Boogaard et al., 1993).

In the present paper, for the sake of generality, no assumptions are made about the model structure and/or about the formulation of the model error function. Also, no assumption is made about the existence of just a single minimum of the error function; the optimization problem is posed as a global optimization problem (GOP).

A global minimization problem with box constraints is considered: find an optimizer $x^{*}$ such that:

$$
\begin{array}{r}
\mathrm{f}^{*}=\mathrm{f}\left(x^{*}\right)=\min \mathrm{f}(x) \\
x \in X
\end{array}
$$

where the objective function $\mathrm{f}(x)$ is defined in the finite interval (box) region of the $n$-dimensional Euclidean space:

$$
X=\left\{x \in \mathrm{R}^{n}: a \leq x \leq b \quad \text { (componentwise) }\right\}
$$

This constrained optimization problem can be transformed to an unconstrained optimization problem by introducing the penalty function with a high value outside the specified constraints. In cases where the exact value of an optimizer cannot be found, one refers to its estimate and, correspondingly, its minimum estimate.

Approaches to solving equations (2)-(3) depend on the properties of $\mathrm{f}(x)$. The following cases may be considered:

- $\mathrm{f}(x)$ is a single- or multi-extreme analytically expressed function;

- $\mathrm{f}(x)$ is a single-extreme function which is not expressed analytically;

- no assumptions are made about the properties of $\mathrm{f}(x)$, so that in the general case it is a multi-extreme function which is not expressed analytically.

In practice, the GOP of calibration is sometimes solved simply by applying singleextremum function minimization (local search). In other problems, where an analytical expression of an objective function is available and derivatives can be computed, gradient-based methods are used (Jacobs, 1977). In the case where no analytical expression is given and derivatives cannot be computed, direct search methods can be used, such as downhill simplex descent (DSD) (Nelder \& Mead, 1965), rotating directions (Rosenbrock, 1960), or direction set method (Powell, 1964). Brent (1973) and Press et al. (1990) describe the modification of Powell's method, where the line minimization is achieved by bracketing and quadratic interpolation. It has been modified for use in constrained optimization problems by the introduction of a penalty function outside the constraints interval, and is used in the GLOBE system which was applied in this study and is described in more detail below.

Many of the engineering applications of the 1970s and 1980s used accepted methods for single-extreme function minimization, but often without the investigation of unimodality. Most researchers recognized the problem of a "good" initial starting point, and even mentioned the necessity of trying several such points, but more rigorous practical procedures were not implemented. This can be partly attributed to the lack of a wider awareness within the engineering community of developments in the area of global optimization. 


\section{APPROACHES TO THE MULTI-EXTREME MINIMIZATION}

The reader is referred to Törn \& ilinskas (1989), Pintér (1995) and Neumaier (1997) for an extensive coverage of various methods. Many algorithms aimed at global search include various ideas, so that no accurate grouping of methods is possible. However, for the purposes of this paper the following groups may be distinguished: set (space) covering techniques; random search methods; methods based on multiple local searches (multistart); evolutionary and genetic algorithms; and other methods (simulated annealing, trajectory techniques, tunnelling approach, analysis methods based on a stochastic model of the objective function).

\section{Set (space) covering methods}

In set covering methods, the search space is covered by $N$ disjoined subsets $X_{1}, \ldots, X_{N}$. Then the objective function is evaluated at $N$ representative points $\left\{x_{1}, \ldots, x_{N}\right\}$ (called a grid), one representing each subset, and an optimizer with the least function value taken as an approximation of a global one. If the way of choosing these sets (or points representing these sets) does not depend on the values that $f$ takes at these points, then the corresponding minimization algorithm is a grid (passive covering) algorithm. In most practical problems such algorithms are inefficient.

However, if all previously chosen points $\left\{x_{1}, \ldots, x_{k}\right\}$ and function values $\left\{\mathrm{f}\left(x_{1}\right), \ldots\right.$, $\mathrm{f}\left(x_{k}\right)$ \} may be used when choosing the next point $x_{k+1}$, then the algorithm is called a sequential (active) covering algorithm (Pinter, 1995). This class of algorithm is preferred to grid algorithms since they provide for the more directed type of search.

If $\mathrm{f}(x)$ is Lipschitzian (i.e. the variation rate of an objective function is bound by a known constant), then the set covering algorithms have some theoretically attractive properties. One of them is the possibility of finding a global minimum with any predefined accuracy, which gives the possibility to establish convergence criteria. In many parameter identification problems, when models exhibit "step-wise" changes in their outputs, the objective function can be non-smooth and even discontinuous; in these cases $\mathrm{f}$ is not always differentiable, the Lipschitz condition does not hold and establishing the convergence properties of algorithms becomes difficult.

\section{Random search methods}

Pure direct random search (uniform sampling) The simplest scheme of obtaining an optimizer assessment, a pure direct random search (pure random search, Monte Carlo search, or direct search) draws $N$ points from a uniform distribution in $X$ and evaluates $f$ at these points; the smallest function value found is the minimum $f^{*}$ assessment. If $\mathrm{f}$ is continuous, then there is an asymptotic guarantee of convergence, but the number of function evaluations grows exponentially with dimension $n$. An improvement is to make the generation of evaluation points in a sequential manner so that the already known function values are taken into account when the next point is chosen, thus producing an adaptive random search.

Controlled random search (CRS) In a controlled random search (Price, 1983), the vector $\mathbf{r}^{k}$ is generated on the basis of a randomly chosen subset of previously 
generated points. The basic idea is that at each iteration a simplex is formed from a sample and a new trial point is generated as a reflection about the centroid of the remaining points; the worst point in the original set is then replaced by the new trial point. The ideas of controlled random search algorithms have been further extended by Ali \& Storey (1994a) producing algorithms referred to as CRS4 and CRS5.

Multistart and clustering The basic idea of the family of multistart methods is to apply a local search procedure several times, and then to make an assessment of the global optimizer. The GLOBE system used in this paper includes two multistart algorithms-Multis and M-Simplex. They are both constructed according to the following pattern:

Step 1 Generate a set of $N$ random points and evaluate the function $\mathrm{f}$ at each of them.

Step 2 (reduction) Reduce the initial set by choosing the $p$ best points (with lowest value of $\mathrm{f})$.

Step 3 (local search) Launch local search procedures starting from each of the $p$ points.

The best point reached is the minimizer assessment.

In Multis, the Powell-Brent local search (Powell, 1964; Brent, 1973; Press et al., 1990) is used at Step 3. M-Simplex is similar to Multis, but instead of Powell-Brent search the downhill simplex descent of Nelder \& Mead (1965) is used.

Popular versions of multistart and other GO algorithms are based on a priori clustering of potential starting points. The region (area) of attraction of a local minimum $x^{*}$ is the set of points in $X$ starting from which a given local search procedure $P$ converges to $x^{*}$. In the ideal case, the multistart methods aim at beginning this local search procedure exactly once in every region of attraction (Törn \& ilinskas, 1989).

Two typical problems are connected with clustering methods. Firstly, clusters may contain several areas of attraction and the local search procedure may miss the global minimum; such a situation can be termed underclustering. Secondly, one region of attraction may be divided over several clusters. In this case the local search procedure will locate the same minimum more than once with corresponding redundant function evaluations; this situation may be called overclustering. The ACCO strategy uses clustering as the first step of randomized search.

Evolutionary algorithms The family of evolutionary algorithms (EA) is based on the idea of modelling the search process of natural evolution, although these models are crude simplifications of biological reality. Evolutionary algorithms introduce some modifications to random search and use terminology from biology and genetics. In genetic algorithm (GA), the binary coding of coordinates is introduced, so that an $l$-bit binary variable is used to represent the integer code of one coordinate $x_{i}$, with the value ranging from 0 to $2^{l}-1$ that can be mapped into the real-valued interval $\left[a_{i}, b_{i}\right]$ (see equation (3)). Connecting the codings of all the coordinates, an overall binary string $G$ of length $n l$ is obtained for each point, which is called the chromosome. For a random sample at each iteration pairs of parent individuals (points) selected on the basis of their "fit" (function value) recombine and generate new "offspring".

Such a recombination (or crossover) operator is defined in the following: select two points (parents) $S$ and $T$ from the population according to some rule (e.g. randomly), select a number $\rho$ (e.g. randomly) between 1 and $n l$, and form either one new 
point $S^{\prime}$, or two new points $S^{\prime}$ and $T^{\prime}$, by taking some of the bits of coordinate values from the first parent $S$, and some from the other parent $T$. The best offspring are selected for the next generation. Offspring may also "mutate"-a randomly chosen bit in the string $G$ is changed to its negative. The idea is that fit parents are likely to produce even fitter offspring. In fact, any random search may be interpreted in terms of biological evolution; generating a random point is analogous to a mutation, and the step made towards the minimum after a successful trial may be treated as a selection.

Back \& Schwefel (1993) give an overview of various evolutionary algorithms, which differ mainly in the types of mutation, recombination and selection operators. Various versions of a canonical genetic algorithm (Goldberg, 1989; Michalewicz, 1996) are applied in many areas (e.g. Wang, 1991; Cieniawski et al., 1995).

Several versions of genetic algorithms were compared (including versions with the "tournament" and "fitness rank" selection) and the one that performed best was chosen for the implementation in the GLOBE system used in the present study. This is a variant of GA with the "fitness rank" selection, one-point crossover, 15-bit coding of variables, bit mutation, and preservation of the best points discovered so far (Solomatine 1999).

\section{THE STRATEGY OF ADAPTIVE CLUSTERING}

The basic ideas behind the adaptive cluster covering (ACCO, and its version, ACCOL) were first outlined by Solomatine (1995) and the details are given in Solomatine (1999). The strategy of ACCO was first conceived as a workable combination of some common sense ideas (reduction, clustering and covering) that could be useful in general-purpose search. The ACCO strategy is based on the following four principles (Fig. 1): clustering (to identify regions of attraction and to launch further global search in them); covering shrinking sub-regions (the values of the objective function are assessed at points drawn from the uniform or some other distribution); adaptation (shifting the sub-region of search, shrinking it, and changing the density of each covering); and periodic randomization (solving the problem several times and/or to rerandomize some populations at intermediate steps).

In Fig. 1 the initial sampling and the regional iterations 1 and 2 for one of the clusters in a two-dimensional case are shown. Depending on the implementation of each of these principles, it is possible to generate a family of various algorithms using non-rectangular domains (hulls), non-uniform sampling and various versions of cluster generation and stopping criteria.

In a variation of ACCO, referred to as ACCOL (adaptive cluster covering with local searches), there are two phases:

ACCO phase ACCO strategy is used to find several regions of attraction, represented by the promising points which are close to potential minimizers (called "potent"). The potent set $P_{1}$ is formed by taking one best point from those found for each cluster during the progress of ACCO. After ACCO stops, the set $P_{1}$ is reduced to $P_{2}$ by leaving only several $m(1, \ldots, 4)$ best points which are also distant from each other.

Local search (LS) phase An accurate algorithm of local search is started from each of the potent points of $P_{2}$ (multistart) in order to find the minimum accurately. In 


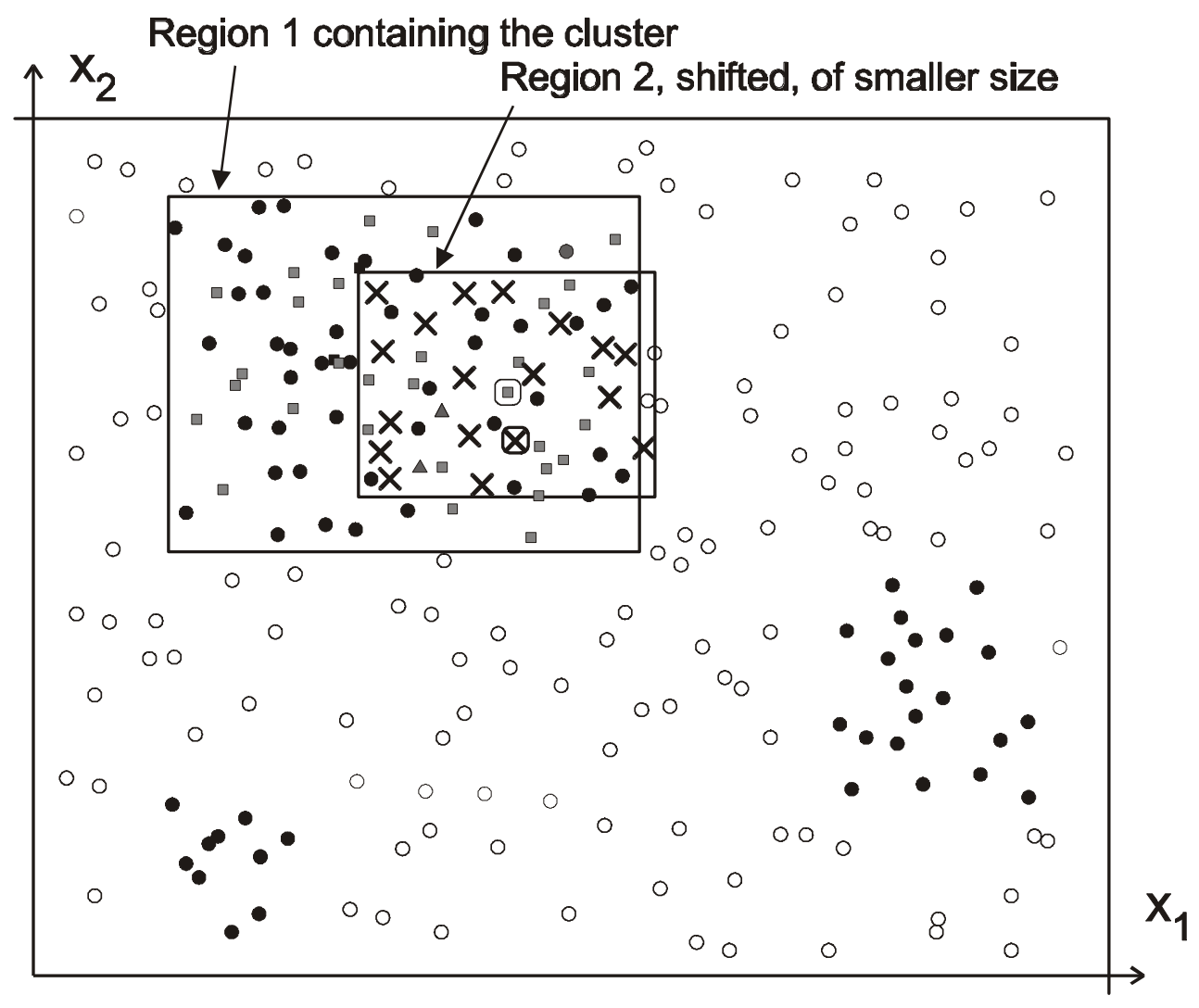

- initial population

- 'good' points grouped into three clusters

- points generated at iteration $1 \times$ points generated at iteration 2

(1) 'best' point in region 1 , around which region 2 is formed

$\otimes$ 'best' point in region 2

Fig. 1 ACCO in a two-dimensional case. Domain is randomly covered, points are evaluated, "good" ones are clustered; for each cluster, smaller regions are repetitively formed around the current "best" point and progressively covered.

the particular implementation, a version of the Powell-Brent search (Powell, 1964; Brent, 1973) was used that does not require the knowledge of gradients.

Experiments have shown that, compared to traditional multistart methods, ACCOL brings significant economy in function evaluations.

\section{GLOBAL OPTIMIZATION TOOL GLOBE}

In order to apply the global optimization techniques to the problems of model calibration, the PC-based system GLOBE has been developed by one of the authors (Solomatine, 1999). GLOBE can be configured to use an external program to supply the objective function values. Figure 2 shows the organization of the calibration process. MODEL must be an executable module (program) which does not require any 


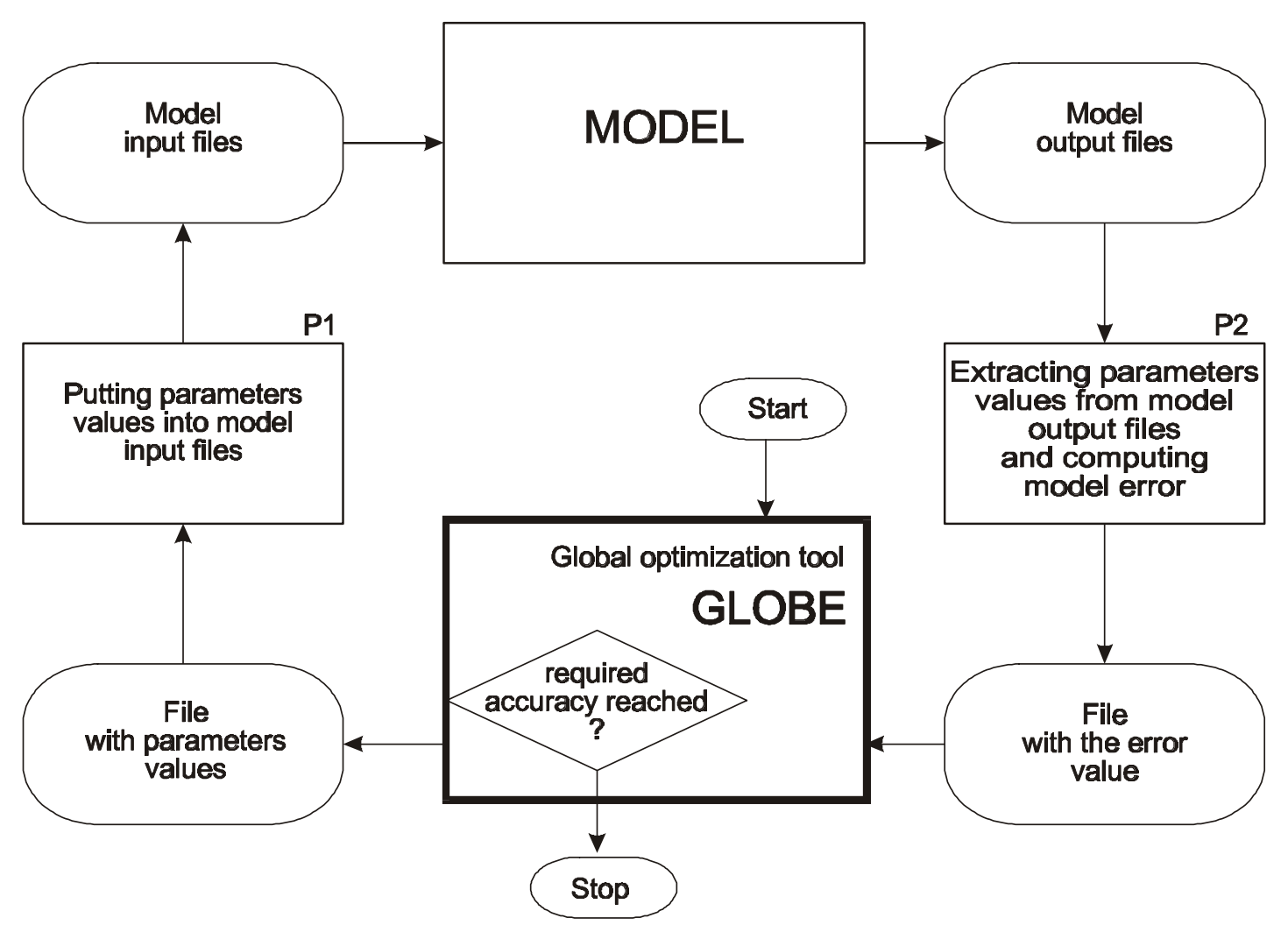

Fig. 2 Organization of the calibration process using GLOBE.

user input, and the user has to supply two transfer programs P1 and P2. These three programs (MODEL, P1, P2) are activated from GLOBE in a loop.

The user interface includes several graphic windows displaying the progress of minimization in different coordinate plane projections. The parameters of the algorithms can be easily changed by the user. Currently, GLOBE includes the following algorithms:

- CRS2 (controlled random search; Price, 1983) described above;

- CRS4a (our modification of the controlled random search by Ali \& Storey, 1994a);

- genetic algorithm (GA), in the version described above;

- Multis, multistart algorithm, as described above;

- M-Simplex, multistart algorithm, as described above;

- $\quad$ adaptive cluster covering (ACCO);

- adaptive cluster covering with local search (ACCOL).

- adaptive cluster descent (ACD) (Solomatine, 1999);

- adaptive cluster descent with local search (ACDL) (Solomatine, 1999).

It is planned to complement GLOBE by such methods as simulated annealing, topographical multilevel single linkage (Torn \& Viitanen, 1994; Ali \& Storey, 1994b) and other reportedly efficient methods.

\section{TRIWACO GROUNDWATER MODEL}

The objective of this study was to investigate the possibility of using global optimization techniques for automatic calibration of a numerical groundwater model of 
the Goeree catchment in The Netherlands. The catchment has been modelled using the TRIWACO package (TRIWACO, 1992).

TRIWACO is a numerical program package for quasi three-dimensional simulation of groundwater flow based on the finite element technique. It has been developed by IWACO BV in The Netherlands, and can be used to build models of groundwater flow in up to nine aquifers, separated by aquitards. The package consists of preprocessing, finite element and post-processing programs. The different program modules of TRIWACO are briefly described below:

Grid generator (TESNET) generates a grid of triangular elements. Rivers, sources and other fixed points are incorporated in the grid. The program is provided with a procedure to generate a more condensed grid around point sources.

Parameter allocator (ARPADI) allocates values of spatially distributed geohydrological parameters (e.g. the transmissivity or hydraulic conductivities) to nodal points in a mesh of triangular elements. These values may be offered to ARPADI in the form of sub-areas, points or a combination of them. This program also allocates values of river or boundary parameters to nodal points.

Finite Element Calculation module (FLAIRS) is a program that performs the finite element calculations for saturated groundwater flow. The program uses triangular elements with linear shape functions. Calculations are based on Galerkin's method and it can handle a variety of problems. The partial differential equation that is (approximately) solved in the program FLAIRS follows from Darcy's law and the continuity equation. The Dupuit-Forchheimer assumption is used, so that the partial differential equations in terms of the potential $h$ (or groundwater head) can be written as:

$$
\frac{\partial}{\partial x}\left[T_{x x} \frac{\partial h}{\partial x}\right]+\frac{\partial}{\partial x}\left[T_{x y} \frac{\partial h}{\partial y}\right]+\frac{\partial}{\partial y}\left[T_{y x} \frac{\partial h}{\partial x}\right]+\frac{\partial}{\partial y}\left[T_{y y} \frac{\partial h}{\partial y}\right]+q=S \frac{\partial h}{\partial t}
$$

For multi-aquifer systems, as in Fig. 3, the above equation holds for each aquifer. The aquifers are coupled by the recharge term $q$ which is the vertical flow through the

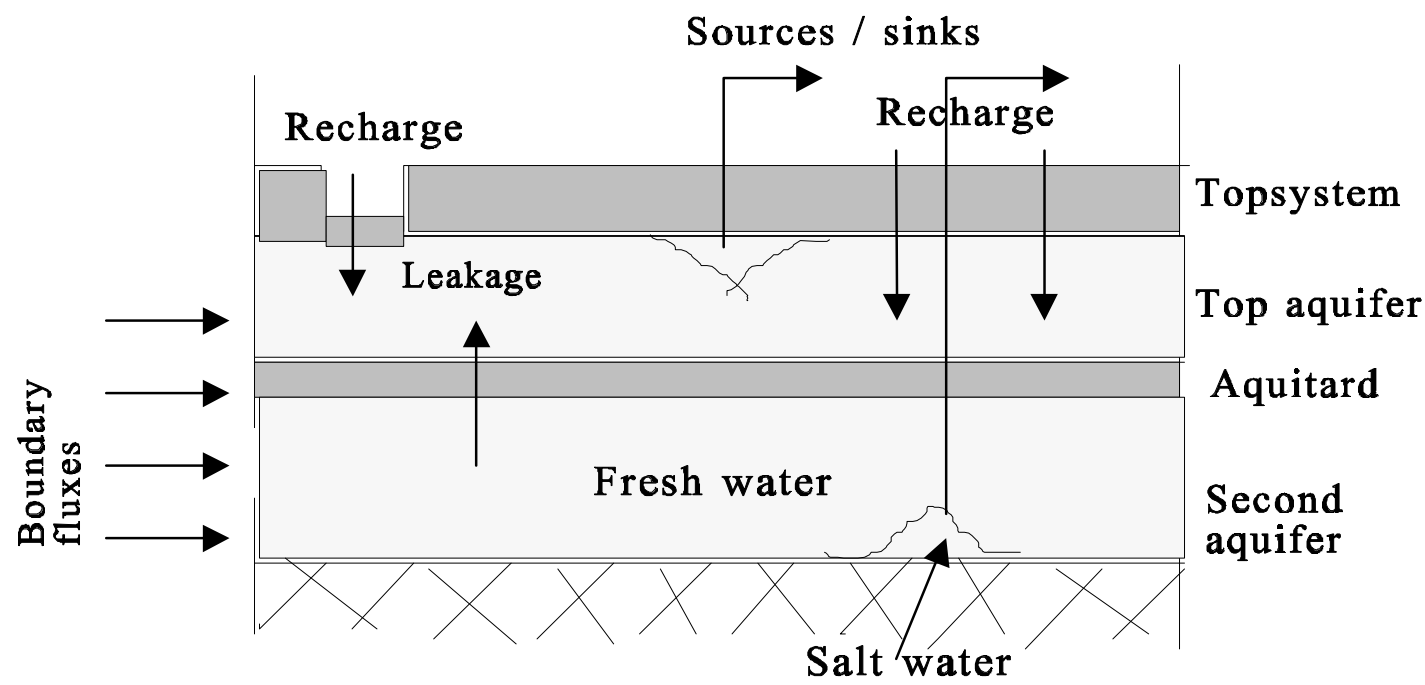

Fig. 3 A multi-aquifer system with recharge terms. 
aquitards. The movement in the horizontal direction is calculated from the heads in the nodes. The vertical movement is determined by means of the continuity condition. The recharge term $q$ describes a number of different effects. In this program it is split into four parts: recharge from a top system due to, for example, precipitation $\left(q_{a}\right)$; leakage through aquitards between aquifers $\left(q_{l}\right)$; point sources or sinks $\left(q_{s}\right)$; and rivers and drains $\left(q_{r}\right)$. The partial differential equation can then be written as:

$$
\frac{\partial}{\partial x}\left[T_{x x} \frac{\partial h}{\partial x}\right]+\frac{\partial}{\partial x}\left[T_{x y} \frac{\partial h}{\partial y}\right]+\frac{\partial}{\partial y}\left[T_{y x} \frac{\partial h}{\partial x}\right]+\frac{\partial}{\partial y}\left[T_{y y} \frac{\partial h}{\partial y}\right]+q_{a}+q_{l}+q_{r}+q_{s}-S \frac{\partial h}{\partial t}=0
$$

The partial differential equation (5) is then solved for the water level $h$ in the different aquifers by the Galerikin finite element method (Huyakorn \& Pinder, 1983).

The stream line and travel time calculation module (TRACE) determines stream lines and travel times of the water particles starting at a user-defined point in the modelled system going upstream or downstream.

Finally, plotting and other post-processing activities allow all the calculation results to be visualized (TRIPLOT module).

\section{TRIWACO model of Goeree}

Goeree is an area located in the southwestern part of The Netherlands; it is a former island in the delta of the rivers Meuse and Schelde. Groundwater is abstracted in the dune area at Kleistee (Fig. 4), while artificial recharge is introduced by a system of drains and infiltration canals present in the dunes.

The groundwater flow system of Goeree has been modelled (Zaadnoordijk, 1990) to gain insight into the current situation and to evaluate different alternatives for increasing the production of drinking water to meet growing demand. Based on the geological information, a system of three aquifers with two aquitards in between, has been identified as shown in Fig. 4. The topmost aquifer is phreatic and consists of sand

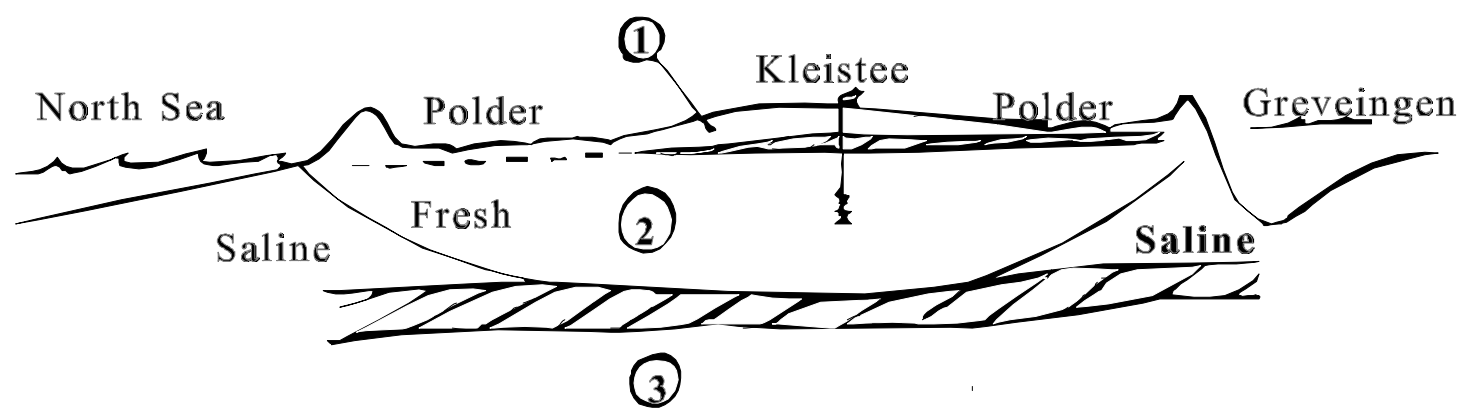

Brackish

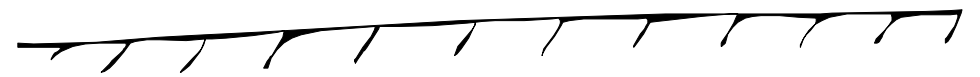

Fig. 4 Aquifer system of Goeree, The Netherlands. 
with a hydraulic conductivity of 5-15 $\mathrm{m} \mathrm{day}^{-1}$, while the second aquifer is composed of sand with a slightly higher hydraulic conductivity ranging between 10 and $20 \mathrm{~m}$ day $^{-1}$. The hydraulic resistance of the first aquitard varies between 600 and 4500 days whereas the that of the second aquitard has values of 1000-15000 days. The third aquifer is almost entirely filled with salt or brackish water and the hydraulic conductivity has an estimated value of $7 \mathrm{~m}^{\text {day }}{ }^{-1}$.

TRIWACO has been used to set up a stationary (steady state) groundwater flow model of Goeree. The model has been calibrated (manually) for the average situation in 1984. Initial values of the parameters were taken from the data survey. They have been refined during the calibration with measured heads in the first and second aquifer.

\section{Parameter identification for calibration}

In the implementation of TRIWACO dozens of parameters are used to specify the geohydrological condition of the groundwater basin. These parameter values are found in the complementary files containing structured data. After careful examination of the groundwater system, three sets of relatively important parameters were selected for the automatic calibration. The other parameters were left as they had been set by the manual calibration. The three sets of parameters were: permeability of the first two aquifers, hydraulic resistance of the semi-pervious top layer (or recharge parameter 2), and resistance of the first aquitard.

Since the parameters are spatially distributed, the total number of parameters involved in each of the above sets are 3,10 and 16 respectively; a total of 29 parameters therefore required calibration. Each parameter describes a particular geohydrological behaviour of a certain region in the groundwater system being modelled.

After identifying the parameters to be optimized, the next task was to specify the range of variability for each parameter. Since there is no detailed information about the geohydrological condition of the study area, the parameter values provided in the manually calibrated model were taken as a reference and a reasonable parameter range around these values was taken for calibration.

Normally, no assumption can be made about the particular nature of the error function corresponding to the different possible combinations of parameters of physically-based groundwater models. In most cases, it is considered as a multiextremum function, which cannot be expressed analytically. The multi-extremum nature of the error function of the model investigated in this study is demonstrated by selecting two of the 29 parameters (permeability and aquitard resistance), and running the model for different combinations of these two parameters $(40 \times 40=1600)$ within their respective ranges while freezing the remaining 27 parameters at predefined values. The corresponding values of the root mean square error (RMSE) between the simulated and observed water levels at the observation wells considered for calibration are plotted in Fig. 5. In addition to the non-smoothness of the error surface, Fig. 5 shows a high number of local minima. If all 29 parameters are allowed to vary, the number of local minima will increase even more. It is obvious that if a local search algorithm is applied in such a situation, it may easily get trapped (attracted) by one of the multiple local minima and will never converge to the global minimum. This justifies the use of global optimization algorithms. 


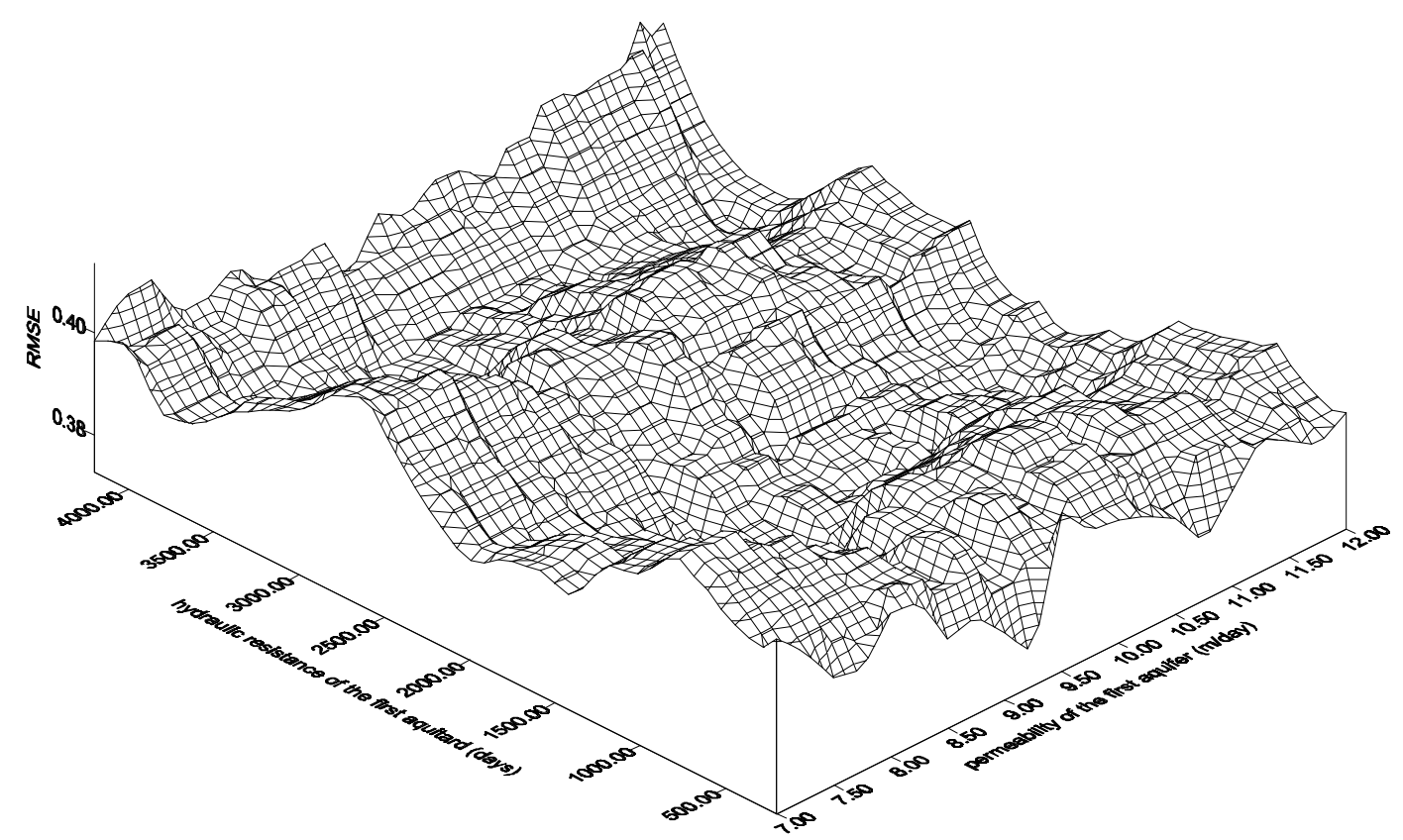

Fig. 5 A typical plot of error function.

\section{CALIBRATION EXPERIMENTS}

TRIWACO was designed as an executable module (program) which does not require any keyboard input, and the two transfer programs P1 and P2 were written (Fig. 2). One run of TRIWACO took several minutes, and one calibration experiment run took up to $16 \mathrm{~h}$ of running time on a Pentium $120 \mathrm{MHz}$ PC. Various GO algorithms were used, but due to the considerable running times, only the performances of ACCO, ACCOL and GA were compared in detail, i.e. with respect to the number of function evaluations needed (efficiency) and accuracy (effectiveness).

In order to obtain a better insight into the calibration process and to access the sensitivity of each group of parameters, the calibration tests were organized in three phases. In the first phase, only the three permeability parameters were considered. During the second phase, all three sets of parameters were considered for calibration and different alternatives were investigated. In the third phase, the performance of the optimization algorithms was investigated with respect to some of their characteristic parameters. The conventional root mean square error (RMSE) between the measured and simulated water levels was used as an objective function. After each calibration phase, the results were presented graphically and interpreted accordingly.

\section{Optimization of three parameters}

In the first phase, the three permeability parameters were considered for calibration. The difference between the simulated and measured water levels are shown in Fig. 6 for both automatic and manual calibration results. The simulated water levels deviate 


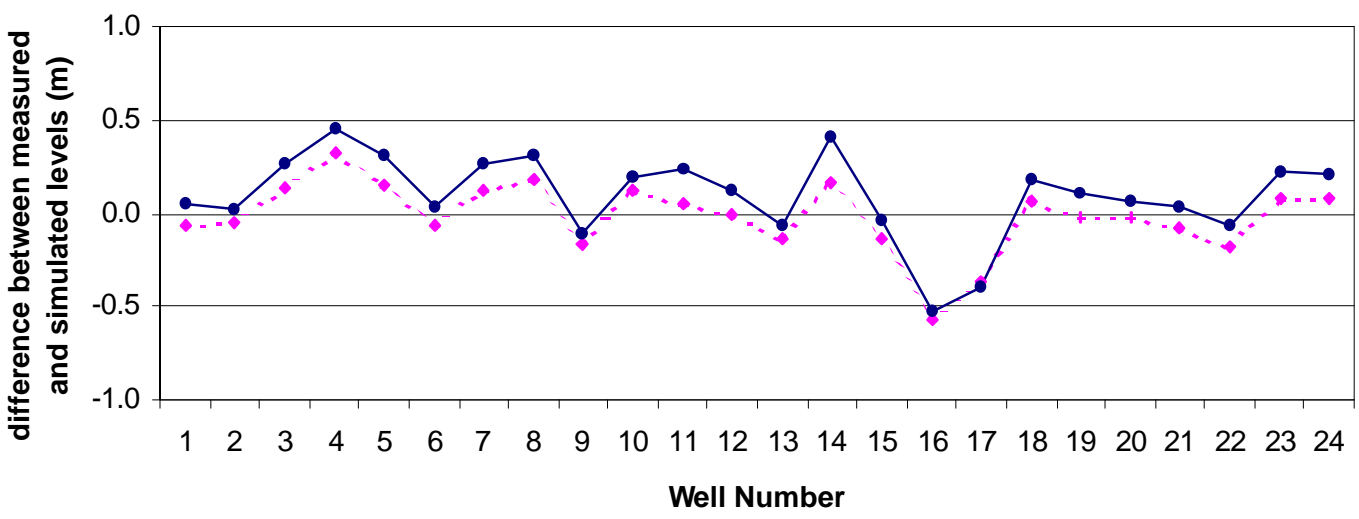

..... ACCO $\longrightarrow$ manual

Fig. 6 Comparison of calibration results: second aquifer, three calibration parameters.

from the observed ones. This might be due to insufficient (i.e. inexact) data about the various boundary conditions in the model. However the automatic calibration results show an improvement over the manual calibration, especially in the second aquifer. A RMSE of 0.40591 was obtained after the manual calibration. ACCO seems to perform better than GA since it achieved the best functional value of 0.368239 at about 645 function evaluations while GA obtained a similar functional value of 0.368511 at about 1170 function evaluations (81\% more than ACCO). A local search launched after ACCO was finished (ACCOL) showed almost no improvement in the RMSE.

\section{Optimization of 29 parameters}

As the number of calibration parameters increased from 3 to 29, a considerable improvement in the error function value was expected. However, no significant improvement was observed (Fig. 7). A closer look at the recharge and resistance

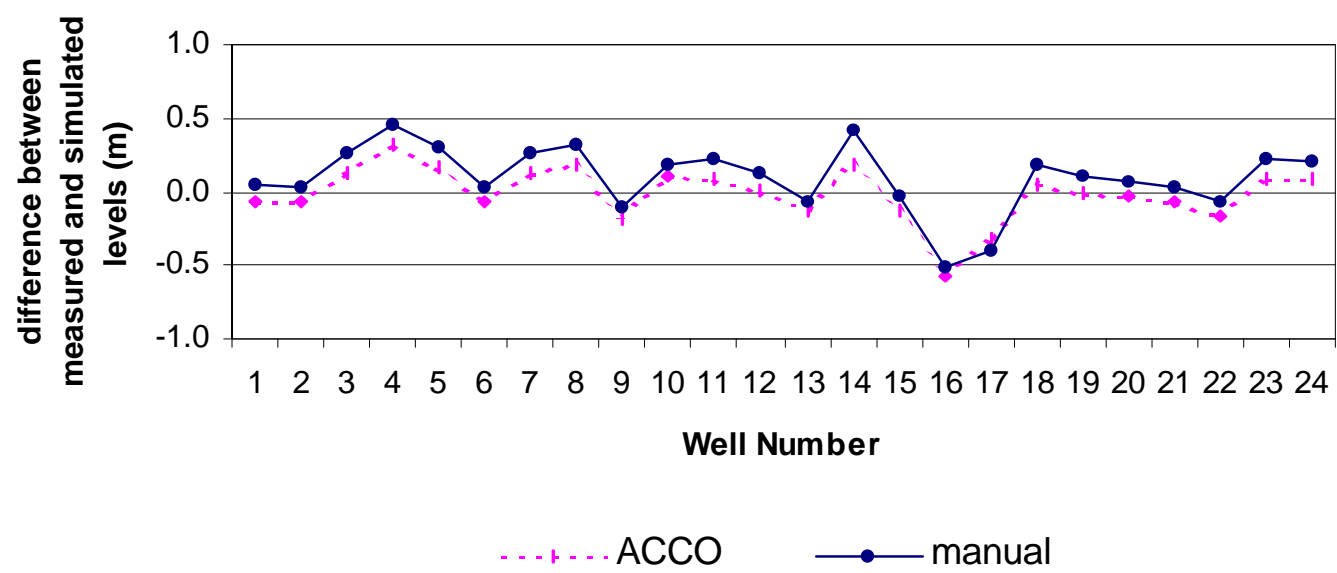

Fig. 7 Comparison of calibration results: second aquifer, 29 calibration parameters. 
Table 1 Summary of calibration results.

\begin{tabular}{|c|c|c|c|c|c|c|c|c|}
\hline \multirow{2}{*}{$\begin{array}{l}\text { Test } \\
\text { no. }\end{array}$} & \multirow{2}{*}{$\begin{array}{l}\text { Optimiz. } \\
\text { algorithm }\end{array}$} & \multirow{2}{*}{$\begin{array}{l}\text { Number of } \\
\text { parameters }\end{array}$} & \multicolumn{4}{|c|}{ Range of parameters values: } & \multirow{2}{*}{$\begin{array}{l}\text { Number of } \\
\text { function } \\
\text { evaluations }\end{array}$} & \multirow{2}{*}{$\begin{array}{l}\text { Minimum } \\
\text { value of error } \\
\text { found }\end{array}$} \\
\hline & & & $\begin{array}{l}\text { PX1 } \\
\left(\mathrm{m} \text { day }^{-1}\right)\end{array}$ & $\begin{array}{l}\text { PX2 } \\
\left(\mathrm{m} \mathrm{day}^{-1}\right)\end{array}$ & $\begin{array}{l}\text { RP2 } \\
\text { (day) }\end{array}$ & $\begin{array}{l}\text { CL1 } \\
\text { (day) }\end{array}$ & & \\
\hline- & Manual & - & - & - & - & - & - & 0.40591 \\
\hline 1 & $\mathrm{ACCO}$ & 3 & $5-15$ & $10-15$ & - & - & 605 & 0.386523 \\
\hline 2 & GA & 3 & $5-15$ & $10-15$ & - & - & 1822 & 0.382884 \\
\hline 3 & $\mathrm{ACCO}$ & 3 & $5-15$ & $10-20$ & - & - & 645 & 0.368239 \\
\hline 4 & GA & 3 & $5-15$ & $10-20$ & - & - & 1170 & 0.368511 \\
\hline 5 & $\mathrm{ACCO}$ & 29 & $5-15$ & $10-15$ & 40 & 400 & 785 & 0.384578 \\
\hline 6 & $\mathrm{ACCO}$ & 29 & $5-15$ & $10-15$ & 60 & 800 & 781 & 0.382623 \\
\hline 7 & $\mathrm{ACCO}$ & 29 & $5-15$ & $10-20$ & 60 & 800 & 791 & 0.36097 \\
\hline 8 & $\mathrm{ACCO}$ & 29 & $5-15$ & $10-20$ & 100 & 1600 & 807 & 0.360555 \\
\hline 9 & $\mathrm{ACCO}$ & 29 & $5-15$ & $10-30$ & 60 & 800 & 775 & 0.35791 \\
\hline 10 & GA & 29 & $5-15$ & $10-30$ & 60 & 800 & 5015 & 0.35171 \\
\hline
\end{tabular}

parameters revealed that in spite of the wider parameter ranges set in the calibration process, the groundwater model is not sensitive to these parameters. The reason for this might be that these parameters have little influence on the behaviour of the groundwater system, or the modelled water levels are not very sensitive to these parameters. The calibration results from both phases of the study are summarized in Table 1.

\section{CONCLUSIONS AND RECOMMENDATIONS}

Even though only limited data sets were tested, the presented experiments with the GLOBE tool (www.ihe.nl/hi/sol/global.htm) show that the automatic calibration of the model, especially with the three permeability parameters, improved results relative to the fully manual calibration.

Due to the considerable running times of hydrodynamic numerical models, the performance of the two optimization algorithms is an important issue. In this particular study both GA and ACCO came up with similar parameter sets and close error function values for given calibration conditions. However, GA required a larger number of function evaluations, and hence, longer running times, than ACCO/ ACCOL. Other experiments (Solomatine, 1999) also confirm the conclusion of the better performance of several global optimization algorithms (ACCO and CRS4) over the canonical genetic algorithm.

In general, once the appropriate parameters for calibration were identified, and some good estimates for the range of parameter values were obtained, the global optimization techniques provided more reliable estimates of the parameters giving the global (rather than local) minimum of the model error.

Automatic calibration should be considered as only a part of the model validation process. The final decisions should be made by experts who have physical insight into the problem and may have to consider factors not included in the model error function.

The following recommendations are made for future studies:

- experiments with groundwater models under transient conditions should be made;

- designers of the modelling codes should foresee the necessity of automatic 
calibration and hence include the ability for programs to run unattended, i.e. without user intervention (Solomatine, 1996);

- due to the considerable running times of groundwater models, another important area for future study is the optimal tuning of different algorithms depending on the class of the problem, the duration of one model run, and the total available time for calibration.

Acknowledgements The authors are grateful to Professors M. B. Abbott, M. J. Hall and R. K. Price for valuable discussions and advice, and the institute IHE-Delft for supporting this research direction. Special words of gratitude go to IWACO BV and particularly to Ir W. J. Zaadnoordijk who provided the groundwater model code and case study used in this research.

\section{REFERENCES}

Ali, M. M. \& Storey, C. (1994a) Modified controlled random search algorithms. Int. J. Comput. Math. 53, $229-235$.

Ali, M. M. \& Storey, C. (1994b) Topographical multilevel single linkage. J. Global Optimiz. 5, 349-358.

Back, T. \& Schwefel, H.-P. (1993) An overview of evolutionary algorithms for parameter optimization. Evolutionary Comput. 1(1), 1-23.

Brent, R. P. (1973) Algorithms for Minimization without Derivatives. Prentice-Hall, Englewood-Cliffs, New Jersey, USA.

Carrera, J. \& Neumann, S. P. (1986) Estimation of aquifer parameters under transient and steady state conditions. 1. Maximum likelihood method incorporating prior information. 2. Uniqueness, stability and solution algorithms. 3. Applications of synthetic and field data. Wat. Resour. Res. 22(2), 199-210, 222-227, 228-242.

Carrera, J. (1988) State of the art of the inverse problem applied to the flow and solute transport equations. In: Groundwater Flow and Quality Modelling (ed. by E. Custodio et al.), 549-583. D. Reidel Publ. Company, Dordrecht, The Netherlands.

Cieniawski, S. E, Eheart, J. W. \& Ranjithan, S. (1995) Using genetic algorithms to solve a multiobjective groundwater monitoring problem. Wat. Resour. Res. 31(2), 399-409.

Dawdy, D. R. \& O’Donnell, T. (1965) Mathematical models of catchment behaviour. J. Hydraul. Div. ASCE 91(HY4), 123-137.

Dixon, L. C. W. \& Szego, G. P. (eds) Towards Global Optimization. North-Holland, Amsterdam, The Netherlands

Franchini, M. \& Galeati, G. (1997) Comparing several genetic algorithm schemes for the calibration of conceptual rainfall-runoff models. Hydrol. Sci. J. 42(3), 357-379.

Goldberg, D. E. (1989) Genetic Algorithms in Search, Optimization and Machine Learning. Addison-Wesley, Reading, Massachusetts, USA.

Hemker, C. J. (1997) Reliability of parameters in automatic calibration (in Dutch). In: Model Calibration, Proc. Conf. Nederlandse Hydrologishe Vereniging), Special issue no. 2.

Huyakorn, P. S. \& Pinder, G. F. (1983) Computational Methods in Subsurface Flow. Academic Press, New York, USA.

Jacobs, D. A. H. (1977) The State of The Art in Numerical Analysis. Academic Press, London, UK.

Johnston, P. R. \& Pilgrim, D. H. (1976) Parameter optimization for watershed models. Wat. Resour. Res. 12(3), $477-486$.

Kuczera, G. (1997) Efficient subspace probabilistic parameter optimization for catchment models. Wat. Resour. Res. 33(1), $177-185$.

Michalewicz, Z. (1996) Genetic Algorithms + Data Structures = Evolution Programs. Springer-Verlag, Berlin, Germany.

Model Calibration (1997) Proc. Model Calibration Conference of the Dutch Hydrological Society. Special issue no. 2., Nederlandse Hydrologishe Vereniging (in Dutch).

Nelder, J. A. \& Mead, R. A. (1965) Simplex method for function minimization. Comput. J. 7(4), 308-313.

Neumaier, A. (1997) http://solon.cma.univie.ac.at/ neum/glopt.html.

Olsthoorn, T. N. (1995) Effective parameter optimization for groundwater model calibration. Ground Water 33, $42-48$.

Peck, A., Gorelick, S., de Marsily, G., Foster, S. \& Kovalevsky, V. (1988) Consequences of Spatial Variability in Aquifer Properties and Data Limitations for Groundwater Modelling Practice. IAHS Publ. no. 175.

Pintér, J. (1995) Global Optimization in Action. Kluwer, Amsterdam, The Netherlands.

Powell, M. J. D. (1964) An efficient method of finding the minimum of a function of several variables without calculating derivatives. Computer J. 7, 155-162.

Press, W. H., Flannery, B. P., Teukolsky, S. A. \& Vetterling, W. T. (1990) Numerical Recipes in Pascal. The Art of Scientific Computing. Cambridge University Press, Cambridge, UK.

Price, W. L. (1983) Global optimization by controlled random search. J. Optimiz. Theory Appl. 40, $333-348$.

Rosenbrock, H. H. (1960) An automatic method for finding the greatest or least value of a function. Comput. J. 3, 175184. 
Scientific Software Group (1996) Product Guide. Scientific Software Group, Washington, USA. See also www.scisoftware.com.

Solomatine, D. P. (1995) The use of global random search methods for model calibration. In: Proc. XXVIth Congress of IAHR (London, September 1995), vol. 1, 224-229. Thomas Telford Ltd., London, UK.

Solomatine, D. P. (1996) Architectures of hydroinformatics systems: pressures form optimization and automatic calibration problems. In: Proc. Second Int. Conf. on Hydroinformatics (Zurich, 9-13 September 1996), 455-460. Balkema, Rotterdam, The Netherlands.

Solomatine, D. P. (1998) Genetic and other global optimization algorithms-comparison and use in calibration problems. In: Proc. Third Int. Conf. on Hydroinformatics (Copenhagen, 24-26 August 1998). Balkema, Rotterdam, The Netherlands.

Solomatine, D. P. (1999) Two strategies of adaptive cluster covering with descent and their comparison to other algorithms. J. Global Optimiz. 14(1), 55-78.

Sorooshian, S., Duan, Q. \& Gupta, V. (1993) Calibration of rainfall-runoff models: application of global optimization to the Sacramento soil moisture accounting model. Wat. Resour. Res. 29(4), 1185-1194.

Törn, A. \& ilinskas, A. (1989) Global Optimization. Springer-Verlag, Berlin, Germany.

Törn, A. \& Viitanen, S. (1994) Topographical Global Optimization using pre-sampled points. J. Global Optimiz. 5, 267276.

TRIWACO Groundwater modelling system (1992). Manual for Version 6.0. IWACO, Rotterdam, The Netherlands.

Van den Boogaard, H. F. P., Hoogkamer, M. J. J. \& Heemink, A. W. (1993) Parameter identification in particle models. Stochastic Hydrol. Hydraul. 7, 109-130.

Wang, Q. J. (1991) The genetic algorithm and its application to calibrating conceptual rainfall-runoff models. Wat. Resour. Res. 27(9), 2467-2471.

Yeh, W. W. (1986) Review of parameter identification procedures in groundwater hydrology: the inverse problem. Wat. Resour. Res. 22(2), 95-108.

Zaadnoordijk, W. J. (1990) An analytic element model of Goeree compared with a finite element model. Poster paper for Model Care 90 Conf. on Calibration and Reliability in Groundwater Modelling (RIVM/IAHS, The Hague, The Netherlands).

Zaadnoordijk, W. J. \& Stein, A. (1997) Objective function and parameters. In: Proc. Model Calibration Conf. of the Dutch Hydrological Society, 21-38. Special issue no. 2, Nederlandse Hydrologishe Vereniging (in Dutch).

Received 4 March 1998; accepted 24 March 1999 